Volume 8. No. 7, July 2020

International Journal of Emerging Trends in Engineering Research

Available Online at http://www.warse.org/IJETER/static/pdf/file/ijeter167872020.pdf

https://doi.org/10.30534/iieter/2020/167872020

\title{
Investigation of the fatigue strength limit of the material
}

\author{
Marina N. Bulaeva ${ }^{1}$, Olga I. Vaganova ${ }^{2}$, Zhanna V. Smirnova $^{3}$, Anna V. Lapshova ${ }^{4}$, Elena A. Chelnokova ${ }^{5}$ \\ ${ }^{1}$ Minin Nizhny Novgorod State Pedagogical University, Nizhny Novgorod, Russia, bulaevamarina@ mail.ru \\ ${ }^{2}$ Minin Nizhny Novgorod State Pedagogical University, Nizhny Novgorod, Russia, vaganova_o@rambler.ru \\ ${ }^{3}$ Minin Nizhny Novgorod State Pedagogical University, Nizhny Novgorod, Russia, \\ z.v.smirnova@mininuniver.ru \\ ${ }^{4}$ Minin Nizhny Novgorod State Pedagogical University, Nizhny Novgorod, Russia, any19.10@ mail.ru \\ ${ }^{5}$ Minin Nizhny Novgorod State Pedagogical University, Nizhny Novgorod, Russia, chelnelena@ gmail.com
}

\begin{abstract}
When solving the problems of ensuring the operability of structures made of composite materials, a significant role is given to non-destructive testing methods that allow you to assess the quality of the product at the stage of manufacture and operation and assess the remaining strength resource. The study of fatigue characteristics allowed us to distinguish two main types of fatigue curves. The article presents the main ideas about the limit of endurance of metal materials.
\end{abstract}

Key words: metals, alloys, fatigue strength, cracks, fatigue curves.

\section{INTRODUCTION}

In recent years, there has been a significant increase in interest in the question of the availability of «the endurance limit of metal alloys. Many aircraft parts and structures must work in the area of gigacycle fatigue. It is assumed that when constructing fatigue curves of metal materials on standard test bases 107-108 loading cycles in semi-logarithmic coordinates» [1]. Basically, you can observe two types of curves. Various types of fatigue curves presented by V. F. Terentiev are shown in figure 1 [1].

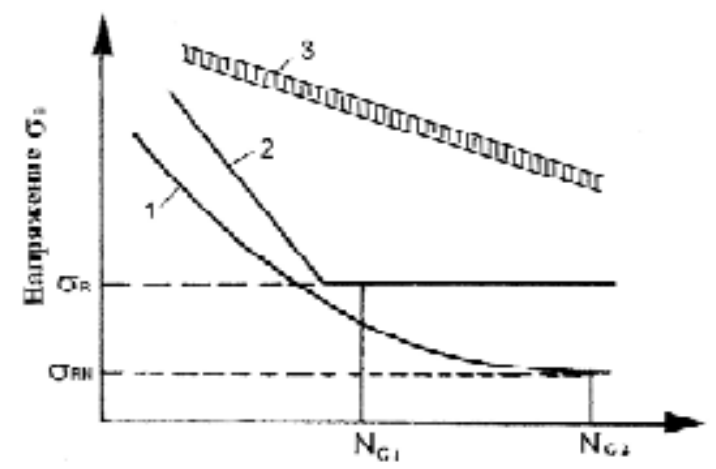

Figure 1: Different types of fatigue curves Horizontal axis - the number of dive cycles $\log \mathrm{N}$.
Where 1 is the fatigue curve for samples with a horizontal section, 2 is the fatigue curve without a horizontal section, and 3 is the straight - line relationship between the stress and the number of cycles. NR1 and NR2 are standard test bases for curves 1 and 2 , respectively.

\section{THEORETICAL FRAMEWORK}

The limit of fatigue (endurance) is «the value of the maximum stress of the cycle, which does not cause the destruction of the sample on the basis of NR. Soft or hard stress-does not matter, since it does not affect the value of the fatigue limit of the material» [3].

The test base is the pre-set number of loading cycles up to which the samples are tested for fatigue [4]. The low level of cyclic loads in the area of fatigue failure causes the appearance of fractures on the surface [5].

The fatigue strength of the metal depends on the composition and properties of the lubricant medium, where the identity of the process of formation and development of cracks in contact-friction and bulk fatigue causes a close influence of the composition of the medium on the fatigue life in both cases [6].

The value of fatigue strength, and therefore durability, is directly dependent on the loading conditions of the structure and the residual stress in them [7].

These are the main factors that affect the durability of structures [8].

The reduction of fatigue strength does not depend on the time of stay of a cyclically loaded part in the medium, nor on the number of loading cycles [9].

When performing the flushing process, the fatigue strength of the edge metal becomes lower by 3-5\% in comparison with the strength of the rolled edge, which eliminates mechanical processing [10]. As a result of eliminating the grooves from cutting and ensuring a more uniform structure of the metal edge, high mechanical properties are achieved [11].

The study shows that the operational spectrum of loads can be systematized [12]. 
Studying the fracture surface without special equipment can reveal the General nature of its structure [13].

Visual inspection is an independent method of fracture analysis that provides valuable data on the quality, causes, and nature of material failure. All features of the structure are applied to the diagram of the fracture and its photo.

The fracture is carefully protected from mechanical damage and oxidation. After examining the fracture, it is cleaned from foreign deposits with a solvent. For this purpose, special combined solvents are used, such as DDV according to GOST 4399-48, P5 according to TU MHP 2191-50, and others). Alcohol and gasoline were used for the same purpose. The oxides present on the fracture of the steel sample are removed by dipping the sample in a $20 \%$ solution of sulfuric acid with the addition of "CHM" according to TU MNP 521-54 for a few minutes.

The world Cup slows down the etching. Fractures of aluminum samples are cleaned in a $10 \%$ solution of alkali $\mathrm{NaOH}, \mathrm{KOH}$.

It is established that the overall durability of samples, parts, or structural elements that worked under cyclic loading with a period of fatigue crack growth, the change of which can occur within $10-90 \%$ of the total durability of the sample or part.

If the structures already have cracks and any other defects, the rate of propagation of the fatigue crack determines the survivability of the construction material [14]. Fatigue failure requires stresses less than the ultimate strength under static loading.

The gradual accumulation of local damages in the metal, which pass into submicroscopic cracks (growing up, they combine into a macroscopic crack) occurs under repeatedly applied cyclic loads. The continued growth of the fatigue crack is controlled by the stress intensity coefficients at its apex [15].

In many cases, the direction of the cracks, which are located near the fracture, serve as an additional sign when establishing the initial zones and the General direction of destruction of the part.

The local stress state of the material at the crack tip and the mechanism of fatigue failure changes as the stress intensity coefficients increase.

The origin of a fatigue crack usually occurs on the surface of samples or parts (the place where the stress from the external load reaches the maximum values).

The origin of cracks can occur on smooth polished surfaces. Their appearance becomes more simple if there is the presence of stress raisers:

- irregularities from mechanical processing;

- inclusions';

- pitting corrosion;

- local wear [16].

This is not an exhaustive list of voltage concentrators.
The origin of a crack under variable bending of a round sample has several stages:

- formation of a crack in the part most loaded, previously subjected to micro-deformations and received maximum hardening;

-then there is a gradual spread of the crack (smooth lapped surface) [16];

- final destruction (doloma zone), there is a decrease in the live section, an increase in the true stress [16].

The calculation of strength at variable stresses was carried out as follows.

First, the calculation of a structural element that is under variable stress begins with a static calculation of the structure, the purpose of which is to determine the dimensions at a given load.

Secondly, the construction of the structure is carried out. Then an updated fatigue test calculation is performed. The safety factor is the ratio of the endurance limit defined for the part to the nominal value of the maximum stress that occurs at the dangerous point of the part during bending and torsion [16].

Due to cyclic loading is the formation of local slip bands and the appearance of the resulting raised areas.

The appearance of such areas is associated with the heterogeneity of the structure of real metals and alloys.

They look like a special type of formation on the surface: - protrusions (extrusion);

- depressions (intrusions) that can be observed in a microscope. Figure 2 shows the bulges and depressions in Nickel(according To G. V. Klevtsov) [16].

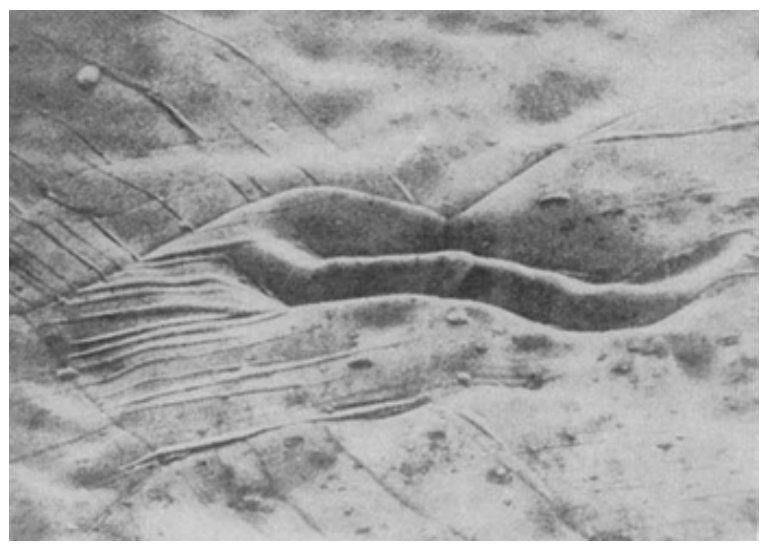

Figure 2: Bulges and depressions in annealed Nickel

Bands of local sliding that lead to the formation of depressions and bulges are formed at approximately an angle of 45 to the direction of the tensile stress (according To G. V. Klevtsov Figure 3). 


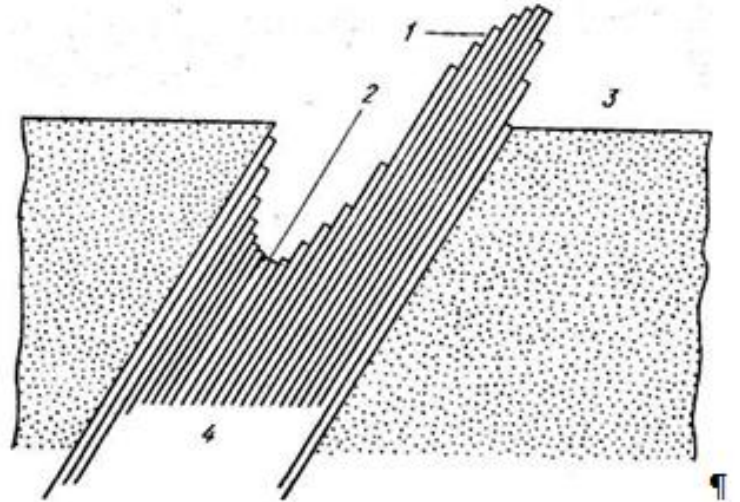

Figure 3: Scheme of formation of extrusions (1) and intrusions (2) on the surface of metal materials subjected to cyclic loads

After the formation of fatigue microcracks, the stage of their propagation occurs [16]. The crack extends perpendicular to the direction of applied stress.

It should be noted the total deformation at fracture: nature of sagnotti the edges of the fracture and the direction of deformation indicate the degree of plastic deformation before fracture and the direction of the effective forces

The number of fatigue zones and their dimensions depend on the number of loading cycles that the samples can withstand until the moment of failure.

The shape of the fatigue zones depends on the configuration of the part and the schemes of applied loads.

At the propagation stage, for each loading cycle, the crack increases forward for a certain period. A number of fatigue grooves appear on the fracture surface (according To G. V. Klevtsov figure 4) [16].

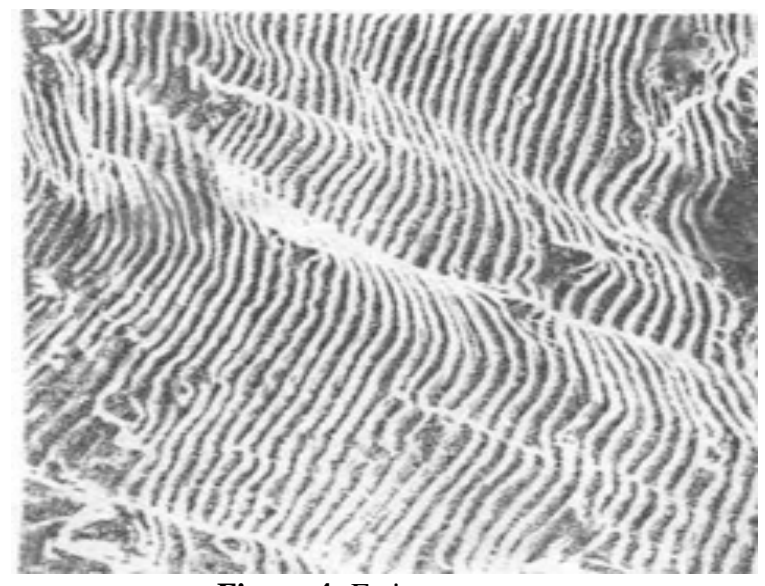

Figure 4: Fatigue grooves

The fatigue curves reveal a stress level that does not lead to the destruction of samples during large loading cycles [16].

\section{RESULTS AND DISCUSSION}

The purpose of the study is to determine the fatigue limit based on the fatigue curve.
The following materials were used for the research:

- fatigue curves of materials;

- set of fatigue fractures that are obtained with low-cycle and multi-cycle fatigue.

The study takes into account loading cycles, the parameters of which are:

- maximum and minimum values of the cycle voltage;

the voltage swing of the cycle;

the frequency and period of loading;

- voltage amplitude;

- the mean stress of the cycle;

- the coefficient of the loading cycle.

For visual and macroscopic study of the fracture was performed in the following order:

- inspection of an untreated fracture with a magnifying glass that magnifies 5-10 times and a binocular microscope at a magnification of 20-40;

- photographing an untreated fracture;

- implementation of flushing, cleaning and drying of the fracture;

- inspection of the cleaned fracture using a magnifying glass that magnifies 5-10 times and a binocular microscope at 20-80 magnification and individual sections of the fracture at 80-120 magnification;

- photographing the fracture and individual sections of its surface.

«The number of micro-damages was determined in real time by a counter based on the count of the number of electromagnetic radiation pulses that were released during the loading of samples» [2].

These parameters are presented by G. V. Klevtsov and shown in figure 5 .

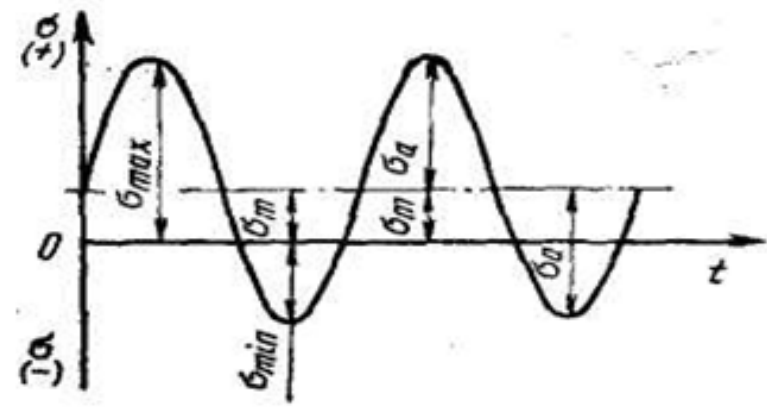

Figure 5: Параметры цикла нагружения

Metal materials that have a physical limit of endurance observed at standard test bases begin to collapse after passing 107-110 loading cycles and a second branch of gigacycle fatigue occurs and a long step between the multi cycle and gigacycle fatigue curve:

- step;

- double;

-multistage fatigue curve. 
The following types of loading were involved in the research: - sign-constant cycles (stretching $0<\mathrm{R}<1$, compressing $1<\mathrm{R}$ ); - alternating cycles (symmetrical $\mathrm{R}=-1$; mainly stretching $(-1<\mathrm{R}<0)$ and mainly compressing $(-\infty<\mathrm{R}<-1)$;

- zero $(\mathrm{R}=0)$, zero compression $(\mathrm{R}=-\infty)$.

At the same time, on large bases of fatigue tests, cracks are more often generated not on the surface, but under the surface layer.

Fatigue curves have two branches of longevity, with a horizontal section between them (the gap of fatigue curves).

The kinetic curves «obtained under cyclic loading conditions differ little in shape from the kinetic curves obtained under loading at a constant speed up to the complete destruction of the samples» [1]. This was due to the number of micro-cracks that were accumulated during the half-loading and half-unloading periods.

\section{CONCLUSION}

We have studied the mechanisms of origin and propagation of fatigue cracks, the structure of fractures obtained during low-cycle and multi-cycle fatigue.

The study of fatigue characteristics allowed us to distinguish two main types of fatigue curves. First, it is fatigue with an ever-increasing durability to failure as cyclic stresses decrease, and second, the fatigue curve with a step-a normalized limit of endurance.

The mechanism of origin of fatigue micro-cracks can be different, however, in plastic metals and alloys that have a plastic phase, it is often associated with intrusions that form in surface stable slip bands.

\section{REFERENCES}

1. V.F. Terentyev. About endurance limit of metal materials. New materials and technology and machine-upgraded. No. 1 pp. 32-38, 2007.

2. T. M. Chernikova, V. V. Ivanov, V.I. Klimov, E. A. Mikhailova. Study of the fatigue strength of materials under cyclic loads. Bulletin of the kuzbass state technical university. No. 2 (46), 73-75, 2005.

3. S. G. Grigoriev, V. A., Shabunina, Ju. M. Tsarapkina, N. V. Dunaeva. Electronic library system as a means of self-development of students of digital generation $\mathbf{Z}$ (on the example of studying the course "Basics of the counselor activity"). Scientific and technical librarie, No. 7. Pp. 78-99. 29. 2019. https://doi.org/10.33186/1027-3689-2019-7-78-99

4. O.A. Andrienko. Network educational technologies and their use when working with students. Humanitarian Balkan Research 2019, 1(3), 5-7. 2019.

5. N.V. Koshechko. Innovations from educational discipline "Pedagogical conflictology" in professional preparation of students. Scientific Vector of the Balkans, 1, 59-63. 2018.
6. Z.V. Smirnova, O.I. Vaganova, O.T. Cherney, E.V. Romanovskaya, N.S. Andryashina, D.S. Toshin, The organization of emergency and dispatching servicing of an apartment house. International Journal of Innovative Technology and Exploring Engineering 8(9), $2019 . \quad$ c. $1477-\quad 1480$ https://doi.org/10.35940/ijitee.I8194.078919

7. Z.V. Smirnova, M.V. Mukhina, O.V. Katkova, M.L. Gruzdeva, O.T. Chernei, Network Interaction as a Factor of Professional Qualities' Development of Service Workers. Lecture Notes in Networks and Systems, 87, pp. 698-704. International Journal of Innovative Technology and Exploring Engineering. https://doi.org/10.1007/978-3-030-29586-8_81

8. G.T. Klinkov The specificity of manifestation of pedagogical communication as a special construct. Scientific Vector of the Balkans, 1, 51-52, 2018.

9. A. A. Sukhikh, T. M., Makhneva, V. B. Dement'ev Austenite in nanostructured maraging steel, Inorganic Materials: Applied Research, vol. 10, no. 4, pp. 966-973. , 2019.

10. Ju.M. Tsarapkina, M.M. Petrova, A.G. Mironov, I.M., Morozova, O.B. Shustova, Robotics as a basis for Informatization of education in children's health camp. Amazonia Investiga, 8 (20). 2019.

11. K.Yu. Boyko, A.V. Skripkina and K.Yu. Smartly. The use of syllabus funds in the technology of time management of self-educational activities of bachelors. Multilevel system of continuous professional education in the socio-cultural sphere: problems of continuity and integration, 73-78. 2019.

12. I.F. Filchenkova, Educational management of innovative activity of teachers as an object of pedagogical research. Vestnik Mininskogo universiteta (Vestnik of Minin University), 2019.7 (4), 3. (in Russ.). 2019.

13. Z.V. Smirnova, A.A. Rudenko, O.I. Vaganova, O.T. Cherney, D.S. Mokerov, E.A. Semakhin, The research of carbon construction steel at laser alloying. International Journal of Innovative Technology and Exploring Engineering. 2019 https://doi.org/10.26795/2307-1281-2019-7-4-3

14. T. K. Andriushchenko. Personal aspects of pedagogue's innovative culture. Scientific Vector of the Balkans, 1, 13-16. 2018.

15. Yu.P. Egorov, Yu.M. Lozinsky, E.I. Marr Laboratory practice materials science for students, students in the field of "Mechanical Engineering", 2015, pp. 34.

16. G.V. Klevtsov. Fractodiagnostics of the destruction of metallic materials and structures: textbook. allowance / G.V. Klevtsov [et al.]. - M.: MI-CC, 2007, pp. 264. 